\title{
Primary extragenital choriocarcinoma in posterior mediastinum in a male adult: a case report
}

\author{
Weigang Zhao ${ }^{1 \#}$, Tangbing Chen ${ }^{2 \#}$, Yi Yang ${ }^{1}$ \\ ${ }^{1}$ Department of Thoracic Surgery, Shanghai Jiao Tong University Affiliated Sixth People's Hospital, Shanghai 200030, China; ${ }^{2}$ Department of \\ Thoracic Surgery, Shanghai Chest Hospital, Shanghai Jiao Tong University, Shanghai 200030, China \\ \#These authors contributed equally to this work. \\ Correspondence to: Yi Yang. Department of Thoracic Surgery, Shanghai Jiao Tong University Affiliated Sixth People's Hospital, Shanghai 200030, \\ China. Email: dryangyi12312@163.com.
}

\begin{abstract}
Primary extragenital choriocarcinoma is a highly malignant tumor and usually occurs in the midline of the body, such as the mediastinum, retroperitoneum, pineal gland and the middle part of the brain. In this report we present a case of primary extragenital choriocarcinoma located in the posterior mediastinum. A 40-year-old man was admitted to hospital with severe chest wall pain for 2 months. Enhanced chest computed tomography (CT) showed a mass of soft tissue measuring $5.2 \mathrm{~cm} \times 4.5 \mathrm{~cm}$ located in the posterior mediastinum. CT-guided percutaneous fine needle aspiration (FNA) biopsy was performed and adenocarcinoma was diagnosed. After careful examination, complete tumor resection with combined resection of part left upper lobe of lung and lymph node dissection were performed. Combining hematoxylin/eosin staining and immunohistochemical a pathological diagnosis of choriocarcinoma was made.
\end{abstract}

Keywords: Primary extragenital choriocarcinoma; male; mediastinum

Submitted Apr 01, 2019. Accepted for publication Aug 29, 2019.

doi: 10.21037/atm.2019.09.42

View this article at: http://dx.doi.org/10.21037/atm.2019.09.42

\section{Introduction}

Choriocarcinoma is a highly malignant tumor originated from syncytiotrophoblast, which can be divided as gestational choriocarcinoma and non-gestational choriocarcinoma (1). For non-gestational choriocarcinoma, testis is the most common primary site while mediastinum only represents a small part (2). Here we report a case of primary extragenital choriocarcinoma detected in the posterior mediastinum in a male adult.

\section{Case presentation}

A 40-year-old man was referred to hospital with chest wall pain for 2 months. The symptom was getting worse gradually and symptoms such as low-grade fever, weight loss were developed along with the time. The patient had not history of malignancy. Chest CT showed a mass of soft tissue about $5.2 \mathrm{~cm} \times 4.5 \mathrm{~cm}$ located in the posterior mediastinum with some adhesion to the upper lobe of left lung. The mass was enhanced unevenly after enhancement (Figure 1). No positive sign was detected in physical examination. After admission, CT-guided percutaneous fine needle aspiration (FNA) biopsy was performed and result suggested adenocarcinoma. No evidence of metastasis was detected in magnetic resonance imaging (MRI) of brain and positron emission tomography (PET) scan. After careful preparation, surgery was performed in March fifth, 2012. Surgical access to the mass was chose as left posterior thoracotomy through the 6th intercostals space in lateral decubitus position. At surgery, we found that the tumor was located in the posterior mediastinum with incomplete capsule while the adherent chest wall and part left up lobe of lung were involved. Complete tumor resection with combined resection of part left upper lobe of lung and involved parietal pleura plus lymph node dissection were 
Page 2 of 4

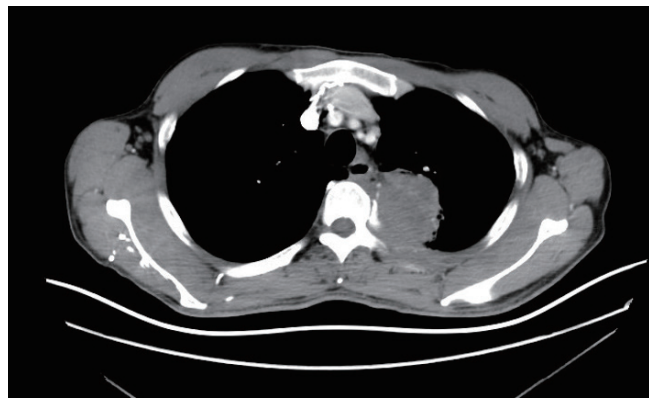

Figure 1 Chest computed tomography before operation.

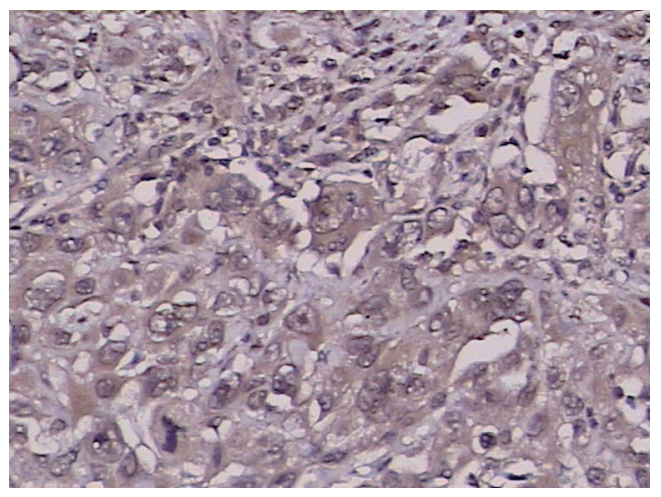

Figure 2 Pathological findings (immunohistochemical results) from the postoperative specimens $(\times 200)$.

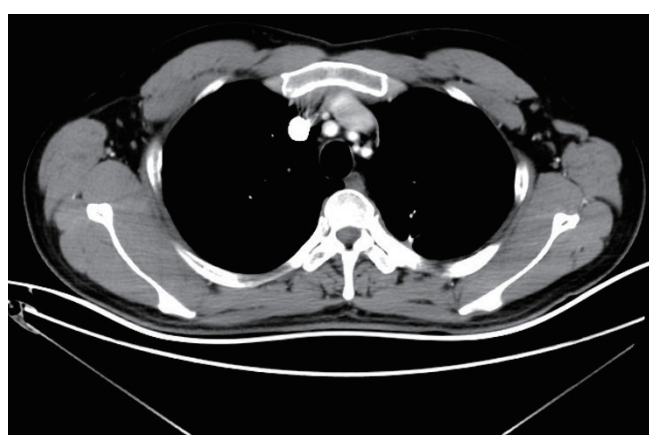

Figure 3 Chest computed tomography after operation.

performed. Microscopic examination showed that atypical cells were arranged in sheets, and syncytial cells were seen. Multiple mitosis and necrosis were detected in tumor cells. Choriocarcinoma comprised of syncytiotrophoblastic and cytotrophoblastic cells. Mediastinal lymph nodes were negative. Immunohistochemical results showed $\mathrm{CK}$ broad (++), CK5/6 (-), P63 (-), 34ßE12 (-), CK7 (-), TTF-1 (-), Naspin (-), S-100 (++), HMB-45 (-), CD56 (-), Syn (-),
Zhao et al. A case of mediastinal primary choriocarcinoma

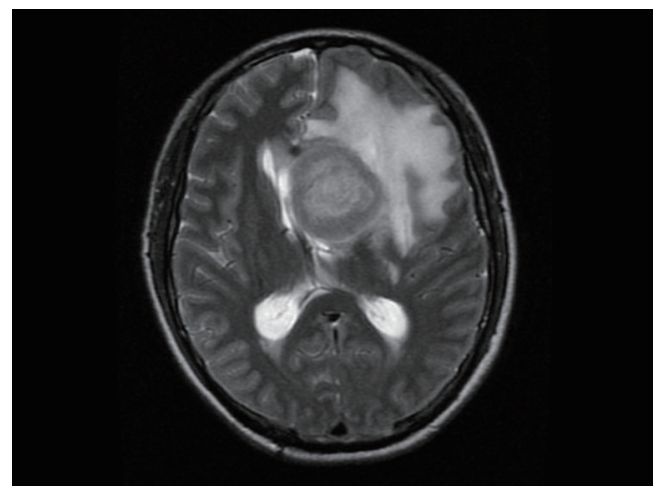

Figure 4 Magnetic resonance imaging (MRI) of brain in 13 months after operation.

CgA (-). Immunohistochemical staining for HCG revealed positive staining in the syncytiotrophoblastic cells of tumor (Figure 2). Combined with hematoxylin/eosin staining and immunohistochemical the diagnosis of choriocarcinoma was made. Enhanced chest CT after surgery (Figure 3) showed tumor was resected completely. Chemotherapy regimen of VP-16 plus cisplatin was given after operation. A total of six cycles of chemotherapy were completed successfully. However, the patients still developed brain metastasis in 13 months after operation (Figure 4) and died in 15 months after operation.

\section{Discussion}

Choriocarcinoma mainly occurs in women after a gestational event and rarely occurs in men as a non-gestational choriocarcinoma (1). Gestational choriocarcinoma originates from the gonads while non-gestational choriocarcinoma occurs in testis, mediastinum, retroperitoneum, pineal gland and the middle part of the brain (2). Primary extragenital choriocarcinoma only presents a small part in non-gestational choriocarcinoma. The prognosis of primary extragenital choriocarcinoma was not optimistic due to the high potential of metastasis while lung was the most common site (3). In this article we presented a case non-gestational choriocarcinoma located in posterior mediastinum in a male adult. Although complete tumor resection and adjuvant chemotherapy were performed, the patients still developed brain metastasis in 13 months after operation and died in 15 months after operation.

The histogenesis of primary extragenital choriocarcinoma is still uncertain. Several theories had tried to explain it and "burned-out tumor" theory was most widely known (4). 
"Burned-out tumor" theory believes that primary extragenital choriocarcinoma is a metastasis from a testicular choriocarcinoma (4). However, no tumor was found in testis during autopsies in several cases contradicted this theory. Another theory believes that tumors originate from retained primordial germ cells that migrate during embryogenesis (5). Thera are still some people believe that tumor arise from non-trophoblastic neoplasm (6). Although above theories have been widely discussed, no conclusion has been reached yet.

Due to the rarity of primary extragenital choriocarcinoma, it is not easy to diagnose this disease preoperatively. In this case, CT-guided percutaneous FNA biopsy was performed and adenocarcinoma was diagnosed. Several reasons may contribute to above result. First, the incidence of pure type choriocarcinoma is rather low while mixed types of choriocarcinoma and adenocarcinoma are more widely seen in clinical work (7). Second, choriocarcinoma is easily misdiagnosed as adenocarcinoma by biopsy due to the small size of the specimen. Third, the rarity of choriocarcinoma in mediastinum makes it difficult for pathologists to diagnose this disease. Compared with FNA, transthoracic CT-guided tru-cut biopsy was preferred for mediastinal tumor with the advantage of providing more pathological tissue for correct diagnosis. However, only a small part of patients were diagnosed correctly via both biopsies preoperatively (8). It is generally accepted that choriocarcinoma can excrete serum $\beta$-human chorionic gonadotropin ( $\beta$-HCG) and $\beta$-HCG is a classic tumor marker for choriocarcinoma $(9,10)$. The lever of $\beta$-HCG levels is usually used as index for evaluating the efficacy of treatment (11). So it was widely tested in patients with choriocarcinoma before and after treatment. Yokoi and colleges even concluded serum HCG test had equal diagnostic value to histopathologic examination (3). However, $\beta$-HCG was not tested preoperatively in this case since choriocarcinoma was not diagnosed before operation, which make it difficult to evaluate the efficacy of surgery according to the HCG lever.

No standard treatment for primary extragenital choriocarcinoma was established (8). Surgery, chemotherapy, radiotherapy, or combinations were the most ordinary treatments for these patients. It is generally accepted surgery is the first choice for operable mediastinal malignant tumors, which is also applicable for mediastinal choriocarcinoma. A study also showed that chemotherapy can significantly prolong the overall survival (OS) of primary extragenital choriocarcinoma (12). In this case, although both surgery and chemotherapy were performed, the patients still developed brain metastasis in 13 months after operation. Stereotactic radiosurgery is now widely used for the treatment of metastatic brain tumors with satisfactory results (13). However, it is only suggested for metastatic tumors less than $3 \mathrm{~cm}$. In this case, stereotactic radiosurgery was not suitable since the size of metastatic brain tumors was more than $3 \mathrm{~cm}$ when detected.

The prognosis of primary extragenital choriocarcinoma is still not optimistic. A study showed that the median OS time was 7.7 months and the 6-month mortality rate was $45.4 \%$ for patients with primary extragenital choriocarcinoma (8). In this case, the patients had survived 15 months, which was longer than most patients in above study. There are several reasons may explain this result. First, the patient was at a relatively early stage before treatment when compared with other patients. Second, completely tumor and invaded tissues resection was performed in this patient. Third, a total of six cycles of adjuvant chemotherapy may prolong the OS.

However, there were still several shortcomings in the course of treatment. First, $\beta$-HCG was not tested before operation. So it is not able to evaluate the efficacy of surgery in this case. Second, postoperative examination of MRI of brain was not performed timely during follow up. Since brain is the common site of metastasis, MRI of brain should be performed routinely instead of performing until symptoms appear. Early treatments of metastatic brain tumors basing on early detection may be able to achieve better result.

In conclusion, primary extragenital choriocarcinoma is a very rare disease with poor prognosis. Early detection and early treatment are very important for long-term survival. Standard treatment for this disease is not established yet. Further research regarding the effective therapy of this disease is wanted.

\section{Acknowledgments}

None.

\section{Footnote}

Conflicts of Interest: The authors have no conflicts of interest to declare.

Etbical Statement: The authors are accountable for all aspects of the work in ensuring that questions related to the accuracy or integrity of any part of the work are 


\section{Page 4 of 4}

appropriately investigated and resolved. Written informed consent was obtained from the patients for publication of this manuscript and any accompanying images.

\section{References}

1. Ngan HY, Bender H, Benedet JL, et al. Gestational trophoblastic neoplasia, FIGO 2000 staging and classification. Int J Gynaecol Obstet 2003;83 Suppl 1:175-7.

2. Moran CA, Suster S. Primary mediastinal choriocarcinomas: a clinicopathologic and immunohistochemical study of eight cases. Am J Surg Pathol 1997;21:1007-12.

3. Yokoi K, Tanaka N, Furukawa K, et al. Male choriocarcinoma with metastasis to the jejunum: a case report and review of the literature. J Nippon Med Sch 2008;75:116-21.

4. Sesterhenn IA, Davis CJ Jr. Pathology of germ cell tumors of the testis. Cancer Control 2004;11:374-87.

5. Fine G, Smith RW Jr, Pachter MR. Primary extragenital choriocarcinoma in the male subject. Case report and review of the literature. Am J Med 1962;32:776-94.

6. Deshpande JR, Kinare SG. Choriocarcinomatous transformation in metastases of an anaplastic lung

Cite this article as: Zhao W, Chen T, Yang Y. Primary extragenital choriocarcinoma in posterior mediastinum in a male adult: a case report. Ann Transl Med 2019;7(22):703. doi: 10.21037/atm.2019.09.42

\section{Zhao et al. A case of mediastinal primary choriocarcinoma}

carcinoma--a case report. Indian J Cancer 1987;24:161-6.

7. Krulewski T, Cohen LB. Choriocarcinoma of the stomach: pathogenesis and clinical characteristics. Am J Gastroenterol 1988;83:1172-5.

8. Jiang F, Xiang Y, Feng FZ, et al. Clinical analysis of 13 males with primary choriocarcinoma and review of the literature. Onco Targets Ther 2014;7:1135-41.

9. Braunstein GD, Vaitukaitis JL, Carbone PP, et al. Ectopic production of human chorionic gonadotrophin by neoplasms. Ann Intern Med 1973;78:39-45.

10. Szturmowicz M, Slodkowska J, Zych J, et al. Frequency and clinical significance of beta-subunit human chorionic gonadotropin expression in non-small cell lung cancer patients. Tumour Biol 1999;20:99-104.

11. Oshima J, Uemura M, Kato T, et al. Modified BEP regimen leads to abatement of choriocarcinoma syndrome in a patient with extra gonadal germ cell tumor : a case report. Hinyokika Kiyo 2014;60:183-7.

12. Kobayashi A, Hasebe T, Endo Y, et al. Primary gastric choriocarcinoma: two case reports and a pooled analysis of 53 cases. Gastric Cancer 2005;8:178-85.

13. Specht HM, Combs SE. Stereotactic radiosurgery of brain metastases. J Neurosurg Sci 2016;60:357-66. 